\title{
Clinical Value Of Apatinib As A Salvage Treatment In Patients With Chemo-Refractory Advanced Cervical Cancer
}

This article was published in the following Dove Press journal: OncoTargets and Therapy

\author{
Meng Su' \\ Ya Gao' \\ XuXue Ye (1D) \\ QingYu Zhou' \\ LiHao Zhao' \\ Xiaona Cai $\mathbb{I D}^{2}$ \\ Didi Chen' \\ Huafang Su' \\ Xiaohua Zhang ${ }^{3, *}$ \\ Congying $\mathrm{Xie}^{\mathrm{l}, *}$ \\ 'Department of Radiotherapy and \\ Chemotherapy, The First Affiliated \\ Hospital of Wenzhou Medical University, \\ Wenzhou, Zhejiang, People's Republic of \\ China; ${ }^{2}$ Derpartment of Ultrasonography, \\ The First Affiliated Hospital of Wenzhou \\ Medical University, Wenzhou, Zhejiang, \\ People's Republic of China; ${ }^{3}$ Department \\ of Thyroid and Breast Surgery, The First \\ Affiliated Hospital of Wenzhou Medical \\ University, Wenzhou, Zhejiang, People's \\ Republic of China
}

*These authors contributed equally to this work

Correspondence: Congying Xie Department of Radiotherapy and Chemotherapy, The First Affiliated Hospital of Wenzhou Medical University, No. 2 Fuxue Street, Wenzhou, Zhejiang 325000, People's Republic of China

Tel +86 |38 677| |88|

Fax +86 57788069372

Email wzxiecongying@।63.com

Xiaohua Zhang

Department of Thyroid and Breast

Surgery, The First Affiliated Hospital of

Wenzhou Medical University, No. 2 Fuxue

Street, Wenzhou, Zhejiang 325000,

People's Republic of China

Tel +86 I 360067 | | 88

Fax +8657755579462

Email zhangxiaohua577@I63.com
Purpose: Apatinib is effective and safe for several advanced or metastatic cancers, but its therapeutic value in cervical cancer is still unknown. The aim of the study was to assess the therapeutic value of apatinib in patients with chemo-refractory advanced cervical cancer.

Patients and methods: This was a retrospective study of patients with advanced cervical cancer treated with apatinib between April 2015 and December 2018 at the First Affiliated Hospital of Wenzhou Medical University, Wenzhou, China. Patients had to have failed at least 2 lines of chemotherapy prior to receiving apatinib. The clinical tumor response was evaluated after 4 weeks of apatinib treatment, and then every 8 weeks (two cycles). Progression-free survival (PFS), overall survival (OS), objective response rate (ORR), disease control rate (DCR), and adverse events were evaluated.

Results: Twenty-five patients were included in this study. The median PFS was 5.8 months (95\% CI, 4.65-6.95), and the median OS was 12.2 months (95\% CI, 8.99-15.41). ORR was $48 \%$ and DCR was $96 \%$. Complete response was not observed. The most common adverse events in this study (all grades) were hand-foot syndrome (48\%), hypertension (20\%), and mouth mucositis $(20 \%)$.

Conclusion: Apatinib monotherapy showed good therapeutic value with tolerable adverse events for patients with chemo-refractory advanced cervical cancer.

Keywords: cervix carcinoma, YN968D1, antiangiogenesis drug, efficacy, safety

\section{Introduction}

Cervical cancer is a growing threat to women's health and remains a main cause of cancer death among women around the world. ${ }^{1}$ Indeed, cervical cancer was estimated to account for more than 12,000 new cancer cases and 4000 deaths in the United States in $2015 .{ }^{1}$ Cervical cancer is the seventh most common cancer among women worldwide. ${ }^{2}$

Patients with cervical cancer often have a favorable response to radical surgery followed by adjuvant therapy (including platinum-based chemotherapy and radiotherapy). ${ }^{3-5}$ Nevertheless, some patients will develop recurrent tumors or will be initially diagnosed as advanced disease. Apart from platinum-resistant cancers, cisplatin or carboplatin plus paclitaxel is an appropriate chemotherapy regimen for these recurrent or advanced tumors. ${ }^{6}$ Despite its effectiveness, the median progression-free survival (PFS) is only 5.3 months. ${ }^{6}$ Furthermore, there is no standard regimen for second- and third-line chemotherapy. After failure of multiple lines of chemotherapy, targeted therapy can be an appropriate choice for 
advanced cervical cancer. ${ }^{7,8}$ Antiangiogenesis therapy is a common choice for these advanced patients.

Bevacizumab is a monoclonal antibody against the vascular endothelial growth factor (VEGF) and has been widely used against chemotherapy-refractory advanced cervical cancer. When adding bevacizumab to chemotherapy, overall survival (OS), PFS, and tumor response can be improved in cervical cancer patients. ${ }^{9}$ Nevertheless, its efficacy is still unsatisfactory. Other VEGF receptor (VEGFR) tyrosine kinase inhibitors (including pazopanib, lapatinib, sunitinib) have been seldom used and showed poor efficacy in patients with cervical cancer. ${ }^{10,11}$

Apatinib belongs to the first generation of oral antiangiogenesis drug and selectively inhibits VEGFR-2, leading to decreased vascular endothelial cell proliferation and migration, and tumor microvascular density. ${ }^{12}$ A Phase II trial in advanced or metastatic gastric carcinoma showed that apatinib could improve OS and PFS compared with placebo, ${ }^{13}$ which was further confirmed by a Phase III trial. ${ }^{14}$ Several papers indicated that apatinib was an appropriate choice for chemo-refractory malignancies. ${ }^{15-17}$

Although some papers have demonstrated that apatinib was effective and safe for advanced or metastatic gastric cancer, breast cancer, ovarian cancer, lung cancer, and liver cancer, ${ }^{13,14,18-22}$ doubts still exist about whether it is an appropriate choice for patients with advanced cervical cancer. Therefore, the aim of this retrospective study was to assess the efficacy and safety of apatinib in advanced cervical cancer patients with $\geq 2$ lines of chemotherapy failure. Although retrospective, this study could suggest novel treatment approach for advanced cervical cancer.

\section{Materials And Methods Study Design And Patients}

This was a retrospective study of patients with advanced cervical cancer treated with apatinib between April 2015 and December 2018 at the First Affiliated Hospital of Wenzhou Medical University, Wenzhou, China. The inclusion criteria were: 1) histopathologically confirmed as cervical squamous cell carcinoma; 2) clinical stage IV; 3 ) had $\geq 2$ lines of chemotherapy failure; 4) received apatinib alone; and 5) received at least one cycle of apatinib. The exclusion criteria were: 1) prior history of other malignancies or 2) prior history of other antiangiogenic therapies.

This study was approved by the Ethics Committee of the First Affiliated Hospital of Wenzhou Medical University,
Wenzhou, China. Written informed consents were obtained from all the patients before apatinib treatment.

\section{Apatinib Treatment}

Apatinib (YN968D1, Jiangsu Hengrui Medicine Co., Ltd., Jiangsu, China) orally administered at an initial dose of 500 mg once daily; if the patient had a body surface $<1.25 \mathrm{~m}^{2}$, the starting dose was $250 \mathrm{mg}$ daily. Then, the dose was adjusted according to adverse events and performance status between $250 \mathrm{mg}$ and $850 \mathrm{mg}$ daily. Dose reduction of apatinib resulting from grade 3-4 adverse events was allowed, but was not lower than $250 \mathrm{mg}$ daily. If no grade 2-4 adverse event was observed, the dose of apatinib could be increased from 250 $\mathrm{mg}$ to $425 \mathrm{mg}$ or $500 \mathrm{mg}$ daily, or $500 \mathrm{mg}$ to $675 \mathrm{mg}$ or $850 \mathrm{mg}$ daily. One treatment cycle was defined as 28 days (4 weeks). All eligible patients received continuous treatment until disease progression, death, or intolerable toxicity (as determined by their physicians).

\section{Assessment Of Clinical Efficacy}

PFS was defined as the duration from starting apatinib to disease progression or death. OS was defined as the duration from starting apatinib to death. The objective response rate (ORR) included complete response (CR) and partial response (PR). The disease control rate (DCR) included $\mathrm{CR}, \mathrm{PR}$, and stable disease (SD).

The clinical tumor response, determined by assessing the degree of tumor shrinkage, was evaluated after 4 weeks of apatinib treatment, and then every 8 weeks (two cycles). Magnetic resonance imaging (MRI) and/or computed tomography (CT) were performed before and during oral apatinib. The tumor response was evaluated using the Response Evaluation Criteria in Solid Tumors (RECIST), version 1.1. ${ }^{23}$ RECIST adopts a unidimensional measurement for solid tumors. CR was defined as the disappearance of all target lesions, and all pathological lymph nodes must have decreased to $<10 \mathrm{~mm}$ in short axis. PR was defined as at least a $30 \%$ decrease in the sum of longest diameters of target lesions, without new lesions. Progressive disease (PD) was defined as at least a $20 \%$ increase in the sum of longest diameters of target lesions and $\geq 5 \mathrm{~mm}$ increase above nadir, or the appearance of new lesions. Patients who did not meet the criteria for PR or PD were classified as having SD.

\section{Clinical Assessment For Toxicity}

Toxicity was analyzed by assessing the incidence of adverse events. The adverse event grades were defined 
according to the Common Terminology Criteria for Adverse Events of the National Cancer Institute, version 3.0 (CTCAE v3.0). ${ }^{24}$

\section{Statistical Analysis}

Descriptive statistics were used. Continuous data were presented as mean \pm standard deviation or median (range). Categorical data were presented as frequencies. Survival analyses, including OS and PFS, were performed using the Kaplan-Meier method. Data management was performed with SPSS 22.0 (IBM Corp., Armonk, NY, USA) and GraphPad Prism, Version 8.0 (GraphPad Software Inc., San Diego, CA, USA).

\section{Results}

\section{Characteristics Of The Patients}

A total of 32 patients with cervical cancer received apatinib alone during the study period, but three were excluded due to treatment duration $<1$ month, four were excluded due to being lost to follow up. Finally, 25 patients were included. The characteristics of the patients are shown in Table 1. Six patients experienced dose adjustment during treatment (Table 2). After dose adjustment, 17 patients (68\%) received $\geq 500 \mathrm{mg} /$ day of apatinib.

\section{Clinical Efficacy}

The survival time and tumor response are presented in Table 3. The median PFS was 5.8 months ( $95 \%$ CI, 4.65-6.95), and the median OS was 12.2 months (95\% CI, 8.99-15.41) (Figure 1). ORR was $48 \%$ and DCR was $96 \%$. Of note, CR was not observed. Decrease of tumor size occurred in 21 patients (84\%) (Figure 2).

\section{Adverse Events}

Table 4 presents the treatment-related adverse events that were observed among all 25 patients. Most adverse events were mild and manageable and there was no adverse event associated with death. The grade $3 / 4$ treatment-related adverse events were hand-foot syndrome $(\mathrm{n}=3,12 \%)$, mouth mucositis $(\mathrm{n}=1,4 \%)$, and thrombocytopenia $(\mathrm{n}=1,4 \%)$. The most common adverse events in this study (all grades) were hand-foot syndrome $(\mathrm{n}=12,48 \%)$, hypertension $(\mathrm{n}=5,20 \%)$, and mouth mucositis $(\mathrm{n}=5,20 \%)$. Dose reduction occurred in five patients due to adverse events. One patient refused oral apatinib after 3 months of treatment, due to grade 4 hand-foot syndrome.
Table I Patient Characteristics

\begin{tabular}{|c|c|}
\hline Characteristics & $n=25$ \\
\hline \multicolumn{2}{|l|}{ Age (years) } \\
\hline Median & 55 \\
\hline Range & $26-73$ \\
\hline \multicolumn{2}{|l|}{ ECOG PS (\%) } \\
\hline 0 & $5(20)$ \\
\hline I & $16(64)$ \\
\hline 2 & $4(16)$ \\
\hline \multicolumn{2}{|l|}{ Stage $^{a}$ at initial diagnosis (\%) } \\
\hline I & $12(48)$ \\
\hline ॥ & $3(12)$ \\
\hline III & $7(28)$ \\
\hline IV & $3(12)$ \\
\hline \multicolumn{2}{|l|}{ Site of metastatic disease (\%) } \\
\hline Nodes & $10(40)$ \\
\hline Lung & $13(52)$ \\
\hline Liver & $4(16)$ \\
\hline Brain & I (4) \\
\hline Pelvic cavity & $7(28)$ \\
\hline Bone & $9(36)$ \\
\hline Soft tissue & I (4) \\
\hline \multicolumn{2}{|l|}{ No. of sites of metastatic disease (\%) } \\
\hline 1 & II (44) \\
\hline 2 & $9(36)$ \\
\hline 3 & $4(16)$ \\
\hline 4 & I (4) \\
\hline \multicolumn{2}{|l|}{ Histological differentiation (\%) } \\
\hline Undifferentiated & $2(8)$ \\
\hline Poorly differentiated & $6(24)$ \\
\hline Moderately differentiated & $17(68)$ \\
\hline \multicolumn{2}{|l|}{ Lines of prior chemotherapy (\%) } \\
\hline 2 lines & $15(60)$ \\
\hline 3 lines & $6(24)$ \\
\hline 4 lines & $4(16)$ \\
\hline \multicolumn{2}{|l|}{ Prior radiation (\%) } \\
\hline Yes & $17(68)$ \\
\hline No & $8(32)$ \\
\hline \multicolumn{2}{|l|}{ Prior radical surgery (\%) } \\
\hline Yes & $19(76)$ \\
\hline No & $6(24)$ \\
\hline
\end{tabular}

Note: ${ }^{a}$ International Federation of Gynecology and Obstetrics 2018 staging. Abbreviation: ECOG PS, Eastern Cooperative Oncology Group performance status.

\section{Typical Case}

A 43-year-old female patient with advanced cervical cancer and with failure to 3 lines of chemotherapy received apatinib at $500 \mathrm{mg}$ daily in September 2015. From September 2015 to June 2016, the longest diameter of the 
Table 2 Dose Adjustment

\begin{tabular}{|c|c|c|}
\hline \multicolumn{2}{|c|}{ Initial Dose (\%) } & $n=25$ \\
\hline \multicolumn{2}{|c|}{$250 \mathrm{mg}$ daily } & $3(12)$ \\
\hline \multicolumn{2}{|c|}{500 mg daily } & $22(88)$ \\
\hline \multicolumn{3}{|c|}{ Dose adjustment (\%) } \\
\hline \multirow[t]{2}{*}{ Yes } & Increase & I (4) \\
\hline & Decrease & $5(20)$ \\
\hline \multicolumn{2}{|l|}{ No } & $19(76)$ \\
\hline \multicolumn{2}{|c|}{ Final dose (\%) } & \\
\hline \multicolumn{2}{|c|}{250 mg daily } & $7(28)$ \\
\hline \multicolumn{2}{|c|}{$425 \mathrm{mg}$ daily } & I (4) \\
\hline \multicolumn{2}{|c|}{500 mg daily } & $16(64)$ \\
\hline \multicolumn{2}{|c|}{675 mg daily } & I (4) \\
\hline
\end{tabular}

Table 3 Efficacy Of Apatinib In Patients With Cervical Cancer

\begin{tabular}{|l|l|l|}
\hline & $\mathbf{n = 2 5}$ & $\mathbf{9 5 \%} \mathbf{~ C l}$ \\
\hline Median PFS (months) & 5.8 & $4.65-6.95$ \\
Median OS (months) & 12.2 & $8.99-15.41$ \\
\hline Tumor response & & \\
PR (\%) & $12(48)$ & \\
SD (\%) & $12(48)$ & \\
PD (\%) & $1(4)$ & \\
\hline ORR & $48 \%$ & \\
DCR & $96 \%$ & \\
\hline
\end{tabular}

Abbreviations: PFS, progression-free survival; OS, overall survival; PR, partial response; SD, stable disease; PD, progressive disease; ORR, objective response rate; $\mathrm{DCR}$, disease control rate; $\mathrm{Cl}$, confidence interval.

retroperitoneal lesion decreased from 33 to $23 \mathrm{~mm}$ and lung metastases also shrunk significantly or disappeared

A

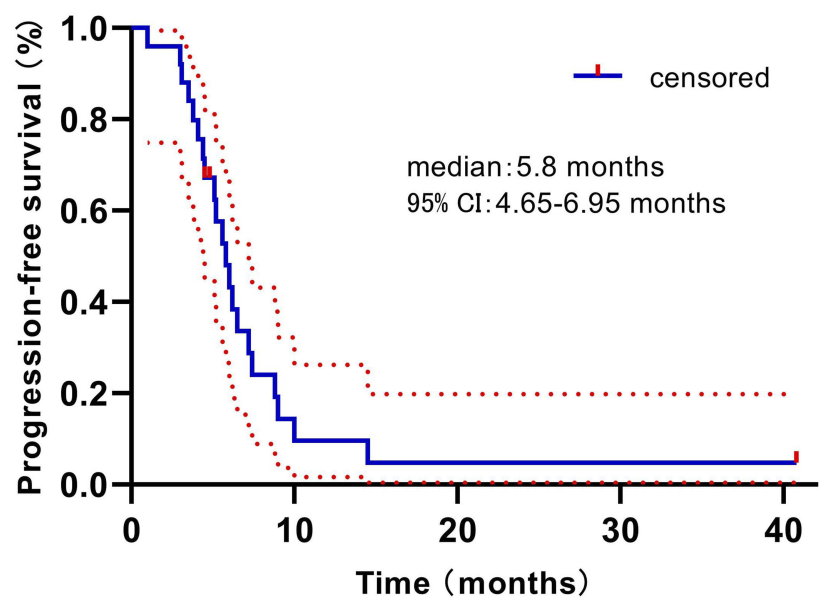

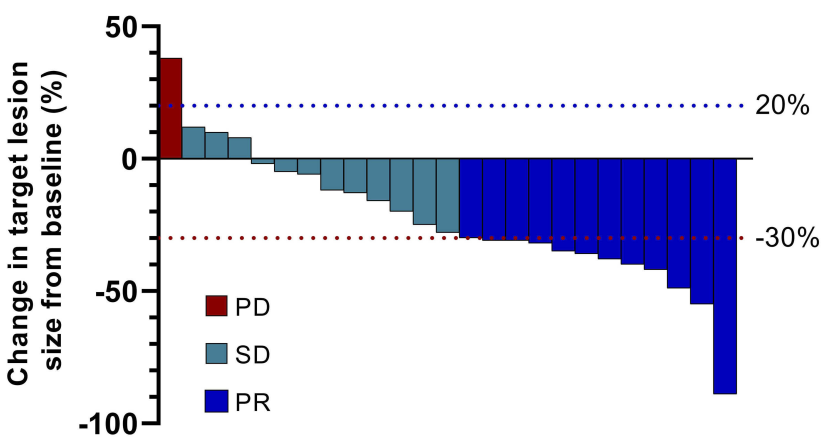

Figure 2 Waterfall plot for the best percentage change in the sum of longest diameters of target lesions from baseline.

(Figure 3), which was defined as PR. Serum squamous cell carcinoma (SCC) antigen decreased from 52.9 to 24.8 $\mu \mathrm{g} / \mathrm{L}$. The patient only suffered from grade 2 hand-foot syndrome. Disease progression occurred on November 2016 and serum SCC antigen increased to $65.7 \mu \mathrm{g} / \mathrm{L}$. Then, the patient stopped to use apatinib.

\section{Discussion}

Cervical cancer is a common female malignancy. The early-staged patients will have favorable outcomes, ${ }^{3-5}$ while advanced ones will have poor prognosis. ${ }^{25}$ Antiangiogenesis therapy is common for advanced cervical cancer, but with limited efficacy. ${ }^{9-11}$ Therefore, to find a new, effective and safe targeted drug for these patients is a matter of urgency for improving treatment outcomes.

VEGF and VEGFR-2 play an essential role in tumor angiogenesis. ${ }^{26}$ Apatinib (formerly known as YN968D1) targets VEGFR-2, RET, platelet-derived growth factor- $\beta$

B



Figure I Kaplan-Meier curve of progression-free survival (A) and overall survival (B). Red-dotted lines represent $95 \%$ confidence intervals (Cls). 
Table 4 Adverse Events Based On Treatment

\begin{tabular}{|l|l|l|}
\hline \multicolumn{2}{|c|}{} & n=25 \\
\cline { 2 - 3 } & Any Grade & Grade 3/4 \\
\hline Hypertension (\%) & $5(20)$ & 0 \\
Proteinuria (\%) & $4(16)$ & 0 \\
Hand-foot syndrome (\%) & I2 (48) & $3(12)$ \\
Mouth mucositis (\%) & $5(20)$ & $\mathrm{I}(4)$ \\
Bleeding (\%) & $\mathrm{I}(4)$ & 0 \\
Fatigue (\%) & $3(12)$ & 0 \\
Abdominal pain (\%) & $\mathrm{I}(4)$ & 0 \\
Decreased appetite (\%) & $2(8)$ & 0 \\
Thrombocytopenia (\%) & $\mathrm{I}(4)$ & $\mathrm{I}(4)$ \\
\hline
\end{tabular}

(PDGFR- $\beta$ ), c-Src, and c-Kit. ${ }^{27,28}$ According to in vitro experiments, apatinib is an even more selective inhibitor of VEGFR-2 than sunitinib, with an IC50 of $0.001 \mathrm{lM}$ and $0.005 \mathrm{lM}$, respectively. ${ }^{12}$ Apatinib can effectively inhibit the proliferation, migration, and tube formation of human umbilical vein endothelial cells, can block the budding of rat aortic ring, and can inhibit the growth of several established human tumor xenograft models with little toxicity. ${ }^{12}$ Previous studies reported that apatinib could reverse ATP-binding cassette transporter (ABC) subfamily B member 1 (MDR1/P-glycoprotein) and ABC subfamily G member 2 (BCRP)-mediated multidrug resistance, which leads to potential usefulness of combining apatinib with other chemotherapy drugs. ${ }^{27,29}$

In a Phase I trial, apatinib has shown encouraging antitumor activity (18.9\% of PR and $64.9 \%$ of SD) and a manageable toxicity profile in patients with advanced solid malignancies, including gastric, colorectal, lung, and breast cancers. ${ }^{30}$ The efficacy and safety of apatinib were further confirmed in advanced or metastatic gastric cancer, breast cancer, ovarian cancer, lung cancer, and liver cancer by recent clinical trials. ${ }^{13,14,18-22}$ These data suggest that apatinib was a favorable choice as rescue therapy for the advanced patients with $\geq 2$ lines of chemotherapy failure. Nevertheless, the therapeutic value of apatinib in cervical cancer is still unknown. In the present study, apatinib showed median PFS, median OS, ORR, and DCR of 5.8 months, 12.2 months, $48 \%$, and $96 \%$, respectively, which were not worse than the data observed in other malignancies. ${ }^{14,18,19,22}$

The efficacy and safety of bevacizumab have been proven by several clinical trials. In a Phase II trial, patients with persistent or recurrent cervical cancer treated with bevacizumab, achieved median PFS of 3.4 months, median OS of 7.3 months, and ORR of $11 \%$ (no CR and five PR, total 46 patients), respectively. ${ }^{31}$ Patients with platinum-resistant cervical cancer can also benefit from bevacizumab. ${ }^{32}$

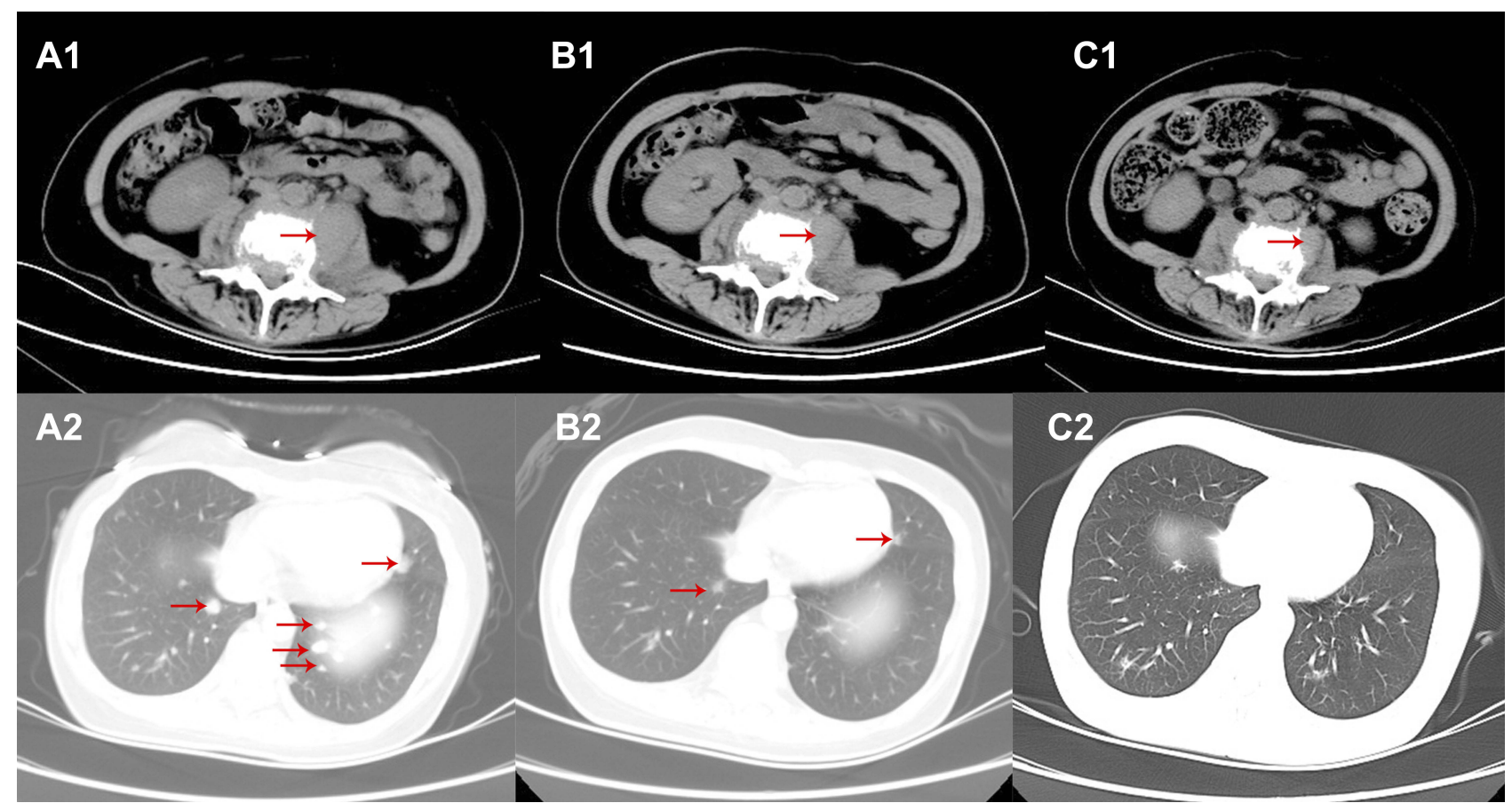

Figure 3 (AI-2) Computed tomography (CT) scan before apatinib treatment (September 20I5). (B I-2) CT scan after I month of apatinib treatment (October 20I5). (CI2) CT scan after 10 months of apatinib treatment (June 20I6). The longest diameters of the retroperitoneal lesions (red arrows) were $33 \mathrm{~mm}$ (AI), $25 \mathrm{~mm}$ (B I), and $23 \mathrm{~mm}$ (C1). From $\mathbf{A} 2$ to $\mathbf{C 2}$, lung metastases (red arrows) shrunk significantly or disappeared. 
Table 5 Comparison Between Apatinib And Other Antiangiogenesis Drugs

\begin{tabular}{|l|l|l|l|l|l|l|l|}
\hline & Lines Of Prior Chemotherapy & $\mathbf{n}$ & mPFS (Months) & mOS (Months) & ORR (\%) & DCR (\%) & Reference \\
\hline Apatinib & $\geq 2$ lines & 25 & 5.8 & 12.2 & 48 & 96 & Present study \\
Bevacizumab & I or 2 lines & 46 & 3.4 & 7.3 & 11 & & 31 \\
Pazopanib & 0, I, or 2 lines & 78 & 18.1 weeks & 50.7 weeks & 9.5 & 52.7 & 10 \\
Lapatinib & 0, I, or 2 lines & 74 & 17.1 weeks & 39.1 weeks & 5.1 & 48.7 & 10 \\
Sunitinib & 0, I, or 2 lines & 19 & 3.5 & & 0 & 84.2 & 11 \\
\hline
\end{tabular}

Abbreviations: mPFS, median progression-free survival; mOS, median overall survival; ORR, objective response rate; DCR, disease control rate; Ref, reference.

Other VEGFR tyrosine kinase inhibitors (including pazopanib, lapatinib, and sunitinib) were seldom used in cervical cancer. In clinical trials of advanced or recurrent cervical cancer, median PFS of pazopanib, lapatinib, and sunitinib were 18.1 weeks, 17.1 weeks, and 3.5 months, respectively; median OS of pazopanib and lapatinib were 50.7 and 39.1 weeks, respectively; ORRs of pazopanib, lapatinib, and sunitinib were $9.5 \%, 5.1 \%$, and $0 \%$, respectively; and DCRs of pazopanib, lapatinib, and sunitinib were $52.7 \%, 48.7 \%$, and $84.2 \%$, respectively. ${ }^{10,11}$ The comparison between apatinib and other antiangiogenesis drugs is shown in Table 5.

The most common treatment-related adverse events of apatinib in this study were hand-foot syndrome (48\%), hypertension $(20 \%)$, and mouth mucositis $(20 \%)$, which were similar to those reported in other malignancies. ${ }^{14,18,19,22}$ During oral apatinib treatment, five patients received dose reductions and one patient discontinued treatment due to treatment-related adverse events. Other patients experienced mild and manageable adverse events.

The present study has two limitations. First, the number of patients was small and clinical significance was limited. The next step will be to further expand the number of patients. Secondly, this was a retrospective study. A multicenter randomized controlled study focusing on this issue is warranted.

\section{Conclusion}

In this study, apatinib monotherapy showed good therapeutic value with tolerable adverse events for patients with chemo-refractory advanced cervical cancer. The feasibility of apatinib combined with chemotherapy in advanced cervical cancer should be further confirmed in future studies.

\section{Abbreviations}

PFS, progression-free survival; ORR, objective response rate; DCR, disease control rate; VEGF, vascular endothelial growth factor; OS, overall survival; VEGFR, vascular endothelial growth factor receptor; CR, complete response; PR, partial response; SD, stable disease; MRI, magnetic resonance imaging; $\mathrm{CT}$, computed tomography; RECIST, Response Evaluation Criteria in Solid Tumors; CTCAE, Common Terminology Criteria for Adverse Events; PD, progressive disease; SCC, squamous cell carcinoma; PDGFR- $\beta$, platelet-derived growth factor- $\beta$; $\mathrm{ABC}$, ATP-binding cassette transporter.

\section{Acknowledgment}

We would like to thank Professor Fengming Kong for her guidance.

\section{Author Contributions}

All authors made substantial contributions to conception and design, acquisition of data, or analysis and interpretation of data; took part in drafting the article or revising it critically for important intellectual content; gave final approval of the version to be published; and agree to be accountable for all aspects of the work.

\section{Funding}

The study is partially supported by funding from Wenzhou Municipal Science and Technology Bureau (2018H0004).

\section{Disclosure}

The authors report no conflicts of interest in this work.

\section{References}

1. Siegel RL, Miller KD, Jemal A. Cancer statistics, 2015. CA Cancer J Clin. 2015;65(1):5-29. doi:10.3322/caac.21254

2. Ferlay J, Soerjomataram I, Dikshit R, et al. Cancer incidence and mortality worldwide: sources, methods and major patterns in GLOBOCAN 2012. Int $J$ Cancer. 2015;136(5):E359-E386. doi:10. 1002/ijc. 29210

3. Falcetta FS, Medeiros LR, Edelweiss MI, Pohlmann PR, Stein AT, Rosa DD. Adjuvant platinum-based chemotherapy for early stage cervical cancer. Cochrane Database Syst Rev. 2016;11:CD005342. doi:10.1002/14651858.CD011360.pub2 
4. Angioli R, Plotti F, Aloisi A, et al. A randomized controlled trial comparing four versus six courses of adjuvant platinum-based chemotherapy in locally advanced cervical cancer patients previously treated with neo-adjuvant chemotherapy plus radical surgery. Gynecol Oncol. 2015;139(3):433-438. doi:10.1016/j.ygyno.2015.09.082

5. Heinzelmann F, Henke G, von Grafenstein M, et al. Adjuvant radiochemotherapy in patients with locally advanced high-risk cervical cancer. Strahlenther Onkol. 2012;188(7):568-575. doi:10.1007/s000 66-012-0123-2

6. Kitagawa R, Katsumata N, Ando M, et al. A multi-institutional phase II trial of paclitaxel and carboplatin in the treatment of advanced or recurrent cervical cancer. Gynecol Oncol. 2012;125(2):307-311. doi:10.1016/j.ygyno.2012.02.009

7. Crafton SM, Salani R. Beyond chemotherapy: an overview and review of targeted therapy in cervical cancer. Clin Ther. 2016;38 (3):449-458. doi:10.1016/j.clinthera.2016.02.007

8. Eskander RN, Tewari KS. Development of bevacizumab in advanced cervical cancer: pharmacodynamic modeling, survival impact and toxicology. Future Oncol. 2015;11(6):909-922. doi:10.2217/fon.14.276

9. Tewari KS, Sill MW, Long HJ 3rd, et al. Improved survival with bevacizumab in advanced cervical cancer. $N$ Engl J Med. 2014;370 (8):734-743. doi:10.1056/NEJMoa1309748

10. Monk BJ, Mas Lopez L, Zarba JJ, et al. Phase II, open-label study of pazopanib or lapatinib monotherapy compared with pazopanib plus lapatinib combination therapy in patients with advanced and recurrent cervical cancer. J Clin Oncol. 2010;28(22):3562-3569. doi:10.1200/ JCO.2009.26.9571

11. Mackay HJ, Tinker A, Winquist E, et al. A phase II study of sunitinib in patients with locally advanced or metastatic cervical carcinoma: NCIC CTG trial IND.184. Gynecol Oncol. 2010;116(2):163-167. doi:10.1016/j.ygyno.2009.08.012

12. Tian S, Quan H, Xie C, et al. YN968D1 is a novel and selective inhibitor of vascular endothelial growth factor receptor-2 tyrosine kinase with potent activity in vitro and in vivo. Cancer Sci. 2011;102(7):1374-1380. doi:10.1111/j.1349-7006.2011.01939.x

13. Li J, Qin S, Xu J, et al. Apatinib for chemotherapy-refractory advanced metastatic gastric cancer: results from a randomized, placebo-controlled, parallel-arm, phase II trial. J Clin Oncol. 2013;31 (26):3219-3225. doi:10.1200/JCO.2013.48.8585

14. Li J, Qin S, Xu J, et al. Randomized, double-blind, placebo-controlled Phase III trial of apatinib in patients with chemotherapyrefractory advanced or metastatic adenocarcinoma of the stomach or gastroesophageal junction. J Clin Oncol. 2016;34(13):14481454. doi:10.1200/JCO.2015.63.5995

15. Xue JM, Astere M, Zhong MX, Lin H, Shen J, Zhu YX. Efficacy and safety of apatinib treatment for gastric cancer, hepatocellular carcinoma and non-small cell lung cancer: a meta-analysis. Onco Targets Ther. 2018;11:6119-6128. doi:10.2147/OTT.S172717

16. Zhao D, Hou H, Zhang X. Progress in the treatment of solid tumors with apatinib: a systematic review. Onco Targets Ther. 2018;11:4137-4147. doi:10.2147/OTT

17. Sun D, Hou H, Zhang C, Zhang X. The efficacy and safety of apatinib for refractory malignancies: a review and meta-analysis. Onco Targets Ther. 2018;11:6539-6554. doi:10.2147/OTT
18. Hu X, Zhang J, Xu B, et al. Multicenter phase II study of apatinib, a novel VEGFR inhibitor in heavily pretreated patients with metastatic triple-negative breast cancer. Int J Cancer. 2014;135(8):1961-1969. doi: $10.1002 /$ ijc. 28829

19. Miao M, Deng G, Luo S, et al. A phase II study of apatinib in patients with recurrent epithelial ovarian cancer. Gynecol Oncol. 2018;148(2):286-290. doi:10.1016/j.ygyno.2017.12.013

20. Lu W, Jin XL, Yang C, et al. Comparison of efficacy between TACE combined with apatinib and TACE alone in the treatment of intermediate and advanced hepatocellular carcinoma: a single-center randomized controlled trial. Cancer Biol Ther. 2017;18(6):433-438. doi:10.1080/15384047.2017.1323589

21. Jiang Q, Zhang NL, Ma DY, Tan BX, Hu X, Fang XD. Efficacy and safety of apatinib plus docetaxel as the second or above line treatment in advanced nonsquamous NSCLC: a multi center prospective study. Medicine (Baltimore). 2019;98(26):e16065. doi:10.1097/ MD.0000000000016065

22. Wu F, Zhang S, Xiong A, et al. A Phase II clinical trial of apatinib in pretreated advanced non-squamous non-small-cell lung cancer. Clin Lung Cancer. 2018;19(6):e831-e842. doi:10.1016/j.cllc.2018.06.002

23. Eisenhauer EA, Therasse P, Bogaerts J, et al. New response evaluation criteria in solid tumours: revised RECIST guideline (version 1.1). Eur J Cancer. 2009;45(2):228-247. doi:10.1016/j.ejca.2008.10.026

24. Trotti A, Colevas AD, Setser A, et al. CTCAE v3.0: development of a comprehensive grading system for the adverse effects of cancer treatment. Semin Radiat Oncol. 2003;13(3):176-181. doi:10.1016/ S1053-4296(03)00031-6

25. Waggoner SE. Cervical cancer. Lancet. 2003;361(9376):2217-2225. doi:10.1016/S0140-6736(03)13778-6

26. Ferrara N. Vascular endothelial growth factor: basic science and clinical progress. Endocr Rev. 2004;25(4):581-611.

27. Tong XZ, Wang F, Liang S, et al. Apatinib (YN968D1) enhances the efficacy of conventional chemotherapeutical drugs in side population cells and ABCB1-overexpressing leukemia cells. Biochem Pharmacol. 2012;83(5):586-597. doi:10.1016/j.bcp.2011.12.007

28. Geng R, Li J. Apatinib for the treatment of gastric cancer. Expert Opin Pharmacother. 2015;16(1):117-122. doi:10.1517/14656566.2015.981 526

29. Mi YJ, Liang YJ, Huang HB, et al. Apatinib (YN968D1) reverses multidrug resistance by inhibiting the efflux function of multiple ATP-binding cassette transporters. Cancer Res. 2010;70(20):79817991. doi:10.1158/0008-5472.CAN-10-0111

30. Li J, Zhao X, Chen L, et al. Safety and pharmacokinetics of novel selective vascular endothelial growth factor receptor-2 inhibitor YN968D1 in patients with advanced malignancies. BMC Cancer. 2010;10:529. doi:10.1186/1471-2407-10-529

31. Monk BJ, Sill MW, Burger RA, Gray HJ, Buekers TE, Roman LD. Phase II trial of bevacizumab in the treatment of persistent or recurrent squamous cell carcinoma of the cervix: a gynecologic oncology group study. $J$ Clin Oncol. 2009;27(7):1069-1074. doi:10.1200/JCO.2008.18.9043

32. Tan SJ, Juan YH, Fu PT, Yu MH, Lai HC. Chemotherapy with low-dose bevacizumab and carboplatin in the treatment of a patient with recurrent cervical cancer. Eur J Gynaecol Oncol. 2010;31(3):350-353.

OncoTargets and Therapy

\section{Dovepress}

\section{Publish your work in this journal}

OncoTargets and Therapy is an international, peer-reviewed, open access journal focusing on the pathological basis of all cancers, potential targets for therapy and treatment protocols employed to improve the management of cancer patients. The journal also focuses on the impact of management programs and new therapeutic

agents and protocols on patient perspectives such as quality of life, adherence and satisfaction. The manuscript management system is completely online and includes a very quick and fair peer-review system, which is all easy to use. Visit http://www.dovepress.com/ testimonials.php to read real quotes from published authors.

Submit your manuscript here: https://www.dovepress.com/oncotargets-and-therapy-journal 